\title{
The difficulties of intrauterine contraceptive research
}

Intrauterine contraception has had a chequered career - from the obscure experiments of Hippocratic times, to the multiplicity of intracervical devices invented by ingenious Victorians, to the gallant but ultimately disappointing experiments of Richter and Grafenberg in the early 20th century. ${ }^{1,2}$ More successful experience came in the 1950 s and 1960s with reports from Ishihama and Oppenheimer. ${ }^{3,4}$ This resulted in a burst of activity from the Population Council of America who organised conferences on intrauterine contraception in 1962 and 1964. . The optimism of those days was summed up by Bernard Berelson, Vice-President of the Population Council, when he said: 'This simple device can and will change the history of the world'. 5

The conferences contained reports from the inventors of new devices ${ }^{6,7}$ resulting in increasing use in various countries including the US, Korea, Taiwan, Chile, Pakistan and Nigeria. ${ }^{8}$ There was a pressing need for serious research.

Of particular significance in the UK was the founding in July 1971 of a Research Unit of the University of Exeter, which has become today the UK Family Planning and Reproductive Health Research Network. The Director was Duncan Mitchell, Professor of Sociology, the Project Director being Robert Snowden, another sociologist. The original aim was to study socio-psychological factors and use-effectiveness of IUDs. This body has continued to organise multicentre research throughout the UK, not only with IUDs. Its latest studies are reported in this issue of the Journal on pages $69-72$.

Over the years there has been the well-known invention of a large number of devices, most of them never actually being used. It almost seems as if any doodler could invent another design! However, many attempts to invent the best possible device have been based on scientific theory. This leads us to the fundamental problem with IUD research. How does one ethically find out if the theory is satisfactory in practice? There are numerous difficulties, some of which are described here, but there isn't space to do other than hint at solutions.

The design of any new device should be based on plausible ideas that aim to maintain the high effectiveness of modern devices while minimising side effects. Initial trials in volunteers require most careful and sensitive organisation.

Trials of a new device and comparative trials of existing devices require large numbers of subjects. This is most easily achieved through multicentre studies which, however, require considerable organisation and control. The results obtained from different centres will vary but combined results have greater validity than those obtained from a single centre.

All clinical trials of course need ethics committee approval, though the audit of established methods does not. It may be helpful if the main centre gets approval that can be quoted to the other ethics committees. Good protocol design is worth every effort to get it right at the beginning so as to avoid confusion and subsequent revisions.

A bugbear of any clinical trial is the phenomena of default. It is impossible to know what events the defaulters may have experienced and which may affect the results. Measures to minimise default should be agreed and be in the protocol.

What about finance? Currently IUD research does not have a very high priority for grants. If finance comes from the manufacturer there must be an understanding that the investigator has complete independence, though the manufacturer will be much involved in the protocol design. It is not enough for the manufacturer to simply supply free devices. Can they pay for the time of clinicians and others who will be involved?

There is often a need to compare results with other published studies. This is not always easy unless identical definitions are used. An example of this problem occurred in the levonorgestrel intrauterine system (IUS) study in this issue of the Journal on pages 73-79. Table 1 shows that the complications of bleeding, pain, amenorrhoea, and hormonal effects have been grouped in various ways making precise comparisons impossible.

Statistical analysis has become very sophisticated these days so there is a need to comply. This will facilitate comparisons and meta-analysis; the difficulties of the latter have been commented on in a recent publication. ${ }^{9}$ The gold standard is the single decrement table described by Tietze $(1973)^{10}$ and by Trussell (1991). ${ }^{11}$

Consideration must be given to confounding factors such as age, parity, socio-economic group, psychosocial aspects, nationality, race, etc. All these factors can affect the results of IUD research.

Apart from research into various devices there is also scope for more research into the mechanism of intrauterine contraception. Also we need to know more about how to improve counselling of clients, the training of personnel, and the provision of services. There is a need to demonstrate with good research, the validity of this method of contraception to parts of the world that are still sceptical, e.g. the US ${ }^{12}$ and Japan. Indeed, a need to counter political, social and commercial obstacles. Last, but not least, we need to know more about how to provide high quality services in developing countries.

We hope this recital of possible problems is not so long as to deter further research; rather our aim is to make future research as valid, effective and useful as possible.

Statements on funding and competing interests

Funding. None declared.

Competing interests. None declared.

Michael L Cox, FRCOG, MFFP

Yew Tree Lodge, Main Street, Higham-on-the-Hill, Nuneaton CV13 6AJ, UK. Tel/Fax: +44 (0) 1455212799

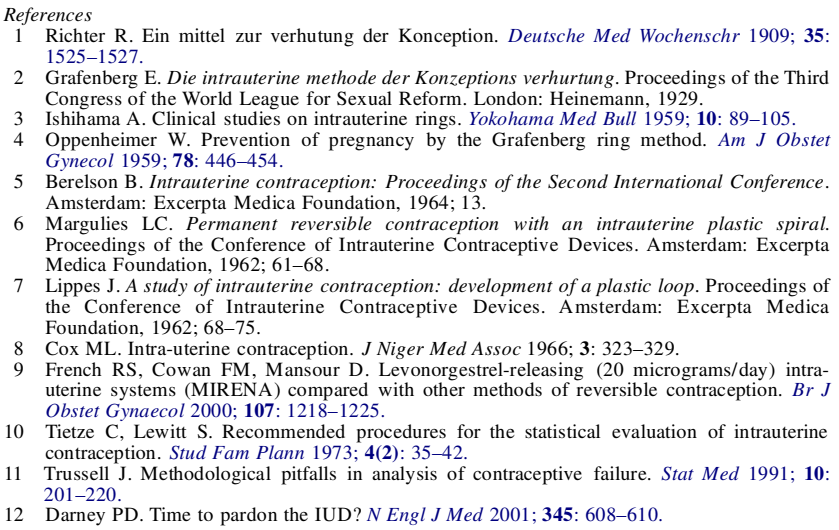
525-1527. cer Konze

Oppenheimer W. Prevetion of pregney by. Proceedings of Deves. Amsterdam: Excerpta Medica

(1960; 3: 323-329. (MTRE) compared with other mothods of reversible contraception. Br J Tetze C, Lewitt S. Recommended poce contraception. Stud Fam Plann 1973; 4(2): 35-42.

Darney PD. Time to pardon the IUD? N Engl J Med 2001; 345: 608-610. 